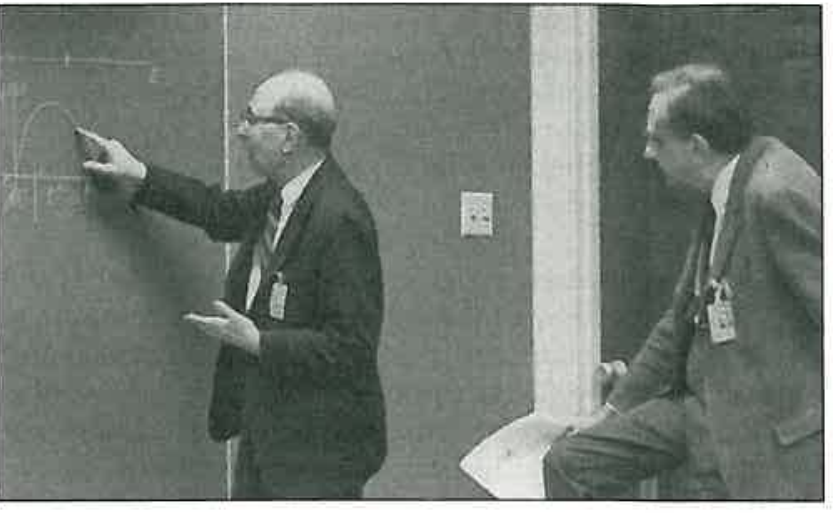

\section{Remembering Eugene}

\section{Wigner and pondering}

\section{his legacy}

Laszlo Tisza, Department of Physics, Massachusetts Institute of Technology, Cambridge, MA, 02139, USA

W hen I looked into the 1992, November issue of Fizikai Szemle in which Wigner was celebrated on his $90^{\text {th }}$ birthday, I saw the list of his well over $\mathbf{3 0 0}$ publications in all branches of physics, in chemistry and in pure mathematics. My first reaction was to withdraw from this attempt of doing him justice in a single talk. On some reflection I thought of a way out.

\section{The early years}

Wigner's life coincided with the $20^{\text {th }}$ century. He was almost of the same "quantum age" as Heisenberg and Pauli, however, these two were in the center of the Copenhagen School and from 1925 on were among the main architects of quantum mechanics $(\mathrm{QM})$. By contrast 1925 was the year when Wigner graduated as chemical engineer in Berlin. He must have felt way behind these pioneers, yet, he soon became one of the leaders of the new discipline. Moreover we shall see that his being rooted in chemistry sheds light on some of the subtler aspects of $Q M$.

Michael Polanyi was among Wigner's mentors in chemistry. Their joint work on molecular reaction chemistry is one of the standard papers in the field. After obtaining his engineering degree Wigner returned to Budapest to work in the tanning factory where his father was director. He felt frustrated, but Polanyi came to the rescue with an invitation to Berlin to an assistantship in $\mathrm{x}$-ray crystallography. Wigner resumed attendance at the physics colloquium and felt great attraction to $\mathrm{QM}$. The factory had been a dead-end, but the chemical training and his sensitization to mathematics in school were positive influences, since $Q M$ was basically a novel confluence of physics, mathematics and chemistry.

Wigner drifted towards physics through a sequence of increasingly purposeful appointments. During this period the relation of

This is an edited version of a talk delivered at the European Physical Society meeting EPS-12 in Budapest, August 26-30, 2002 the just mentioned disciplines changed radically and I will focus on Wigner's contribution to this change.

\section{From chemistry to physics}

Let me remind you of the status of chemistry at the time. The critical role of nitrogen fixation for the Central Powers' ability to pursue World War I was well known. Fritz Haber was awarded the chemistry Nobel Prize in 1918 for this achievement. At the time mathematics and physics did not seem like practical careers. The
$\$$ Photo: Wigner at the

blackboard with Teller. fathers of John v. Neumann and Edward Teller also directed their sons toward chemical engineering. Yet, all three moved from chemistry that seemed to them an empirical craft, toward a physics based on mechanics that was already penetrated by subtle mathematics. An alternative way to see this is that chemistry changed from being an empirical craft to a discipline increasingly intertwined with mathematical physics.

Wigner made considerable contributions to this process. His excellent chemical engineering training in Berlin prepared him for his role of designing the plutonium production facility in the Manhattan Project. The task was to upgrade traditional chemical engineering techniques to include nuclear phenomena, say, the novel cooling problems. This cooperative effort with chemical engineers turned Wigner into a pioneer of nuclear engineering.

Wigner's background helped shape his contribution to fundamental QM years before this event. His early experience in $\mathrm{x}$-ray crystallography called his attention to symmetry. This resonated with his liking for mathematics stimulated by a favorite high school teacher Dr. Laszlo Ratz. His friend Johnny von Neumann substantially added to this orientation. All this culminated in a program of applying the theory of group representations to atomic spectroscopy. The papers that he wrote in 1927-9, some of them jointly with Neumann, are seminal in the field.

At that time most physicists disliked group theory, a sentiment expressed in the widely used "Gruppenpest". This parlance was not a whimsical expression of distaste, but had a philosophical background. Most classical physicists expected infinitesimal analysis to be the natural mathematics for all of physics, with priority accorded to the differential equations of Newtonian mechanics. It was a widely held tacit assumption that this must be the way mathematics enters microphysics. One of the reasons that QM is still not accepted with complete ease is that its most appropriate way to mathematics is different. Such a new way is provided by group theory. Although the important rotation group is continuous, the theory of group representations deals with a discrete substructure. It was Johnny von Neumann who alerted Wigner to this highly esoteric link between discrete and continuous mathematics and one of the non-Newtonian entry ports for mathematics into QM. This effort culminated in the book Group Theory Application to the QM of Atomic Spectra, 1931. This work in German was translated into English in 1959 and appeared in many editions. 


\section{The Nobel Prize}

Although Wigner's book was confined to atomic spectroscopy, he authored group-theory papers also on molecular spectra, solid state, nuclear physics and the infinite unitary representations of the Lorentz group. His contribution to symmetries, particularly in the context of nuclear physics was awarded the

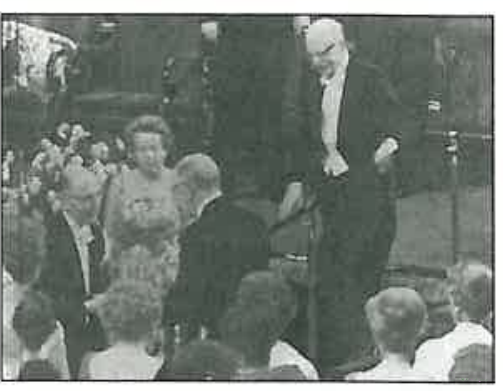

$\triangle$ Photo: The Award of the Nobel Pl
Nobel Prize in 1963. My lack of competence in nuclear physics and the vast number of papers keeps me from highlighting his principal achievements along these lines. However, it is not hard to hint how could Wigner make reliable contributions to so many subdisciplines of physics? His oeuvre was centered on chemistry made up of a hierarchy of levels: structure of atoms, molecules, crystals and nuclei and to some extent elementary particles. A precise and reliable mathematical description was given in terms of group theory. This vast collection of papers constitutes the bulk of Wigner's legacy. Yet there is something else. QM has mysterious paradoxical aspects and there is no unanimity even as to the definition of the difficulties, let alone as to their removal.

Whereas the rules of experimental precision and mathematical rigor are well established, I believe that the rules for associating mathematics with experience are sufficiently ambiguous to give rise to paradoxes. This seldom-featured ambiguity is the butt of Wigner's ironical musings in The Unreasonable Effectiveness of Mathematics in the Natural Sciences. (Comm. in Pure and Appl. Math. 13, No.1, 1960; reprinted in Wigner, Symmetries and Reflections, Indiana University Press, Bloomington \& London, 1967, p 222.)

This is an often reproduced and widely read paper; it has great charm with an understated sense of humor. It is utterly free of technical jargon, but has a complex message, the first part of which is that "mathematics is effective in the natural sciences". This message is undisputed but it is not new. It is more questionable why this effectiveness should be "unreasonable"? In the biography by Andrew Szanton to which Wigner generously contributed, a word count would seem to reveal the importance he assigned to what is "reasonable". What should we make of the prominent use of "unreasonable" in the title of this paper?

\section{Unreasonable theory}

A theory will be deemed "unreasonable" if it conflicts with common sense imbedded in established tradition. Under such conditions one will place a new theory "on probation". There were many theories that were emended or have fallen by the wayside and are happily forgotten. It is more interesting that occasionally "unreasonable" theories are stubbornly irrepressible. Think of the unreasonably moving earth of Copernicus.

After the new theory is sufficiently confirmed, it appeared that it is "common sense" that has to be harmonized with it, rather than vice versa. Nothing is so hard as modifying entrenched tradition and it is remarkable that mathematical physics excels in handling such situations. The heuristic foundation is then reclassified as temporary scaffolding that only hides the beauty of the façade. We can make use of the contrasting role of the scaffolding only if we recognize science as a two-stage process. In the heuristic stage the prime concern is to extend the frontier even at the price of contradiction and paradox. In the stage of consolidation, or rational reconstruction all paradoxes must be resolved and entrenched prejudices abandoned: the cathedral stands even as the scaffolding is removed. It is an entirely novel insight of scientific methodology that the logical standards in the two stages are very different.

Wigner was and remained ambivalent as to this issue, but we can filter out two inconsistent lines within his argument and examine the condition under which they could be reconciled with each other.

\section{Quantum mechanics}

Wigner sees that the superior qualities of QM are unaffected by the flaws of its foundations. He concludes on a cheerful note: "The miracle of appropriateness of the language of mathematics for the formulation of the laws of physics is a wonderful gift which we neither understand nor deserve. We should be grateful for it and hope that it will remain in future research and that it will extend, for better or for worse, to our pleasure, even though to our bafflement, to wide branches of learning."

This is a "cheerful note" in the sense that it comforts the pioneer who was desperate to establish a new bridgehead even if he had to violate "common sense". There was a time span of almost a century and a half between the masterpieces of Copernicus and Newton. The transitional figures of this period achieved their role only because they were able to operate in a logical twilight zone of contradictory notions. From the point of view of distant descendents the most interesting lesson of history is the struggle from the twilight zone into clarity by overcoming inherited dogma, the generator of paradox. This struggle can be turned into a paradigm that was to be replayed in a few instances.

The outstanding example of the $20^{\text {th }}$ century is Einstein's special relativity (SRT). The significant interesting lesson difference from the Lorentz-Poincare theory is Einstein's insight that of history is the Newtonian absolute time has only asymptotic validity. This followed struggle from the from the postulated consistency of twilight zone Maxwellian electrodynamics.

Einstein recognized that the methodology of SRT is superior to that of QM. Although SRT strains our common sense notion of absolute time, Einstein taught us how to do without this notion for uncommon experiences.

By contrast, we haven't yet learned to recognize the provincialism we have to keep under control to be comfortable with QM. According to a well-reasoned recent study, this discipline is no less paradoxical than ever.(See F. Laloë, Am. J. Phys, 69, 2001, p 655.)

When Wigner was first introduced to $Q M$, paradoxes were rampant; yet the theory was utterly convincing; hence Wigner's acceptance of "unreasonable" methods. When he wrote this paper thirty years later, he had a secret hope that a sharpened method might lead to the resolution of paradoxes. I believe this secret hope may have been behind the mysterious motto of the paper: "... and it is probable that there is some secret here which remains to be discovered." - C. S. Peirce.

\section{Experience and mathematics}

What else could be the "secret", but a rational explanation of the "unreasonable" correspondence between experience and 
mathematics. The apparent conflict between the two attitudes can be reconciled by realizing that the scientific enterprise is a composite of apparently opposite moves. The first heuristic penetration into a new area of knowledge calls for the free creative imagination of the discoverer, maybe defying common sense The second stage of consolidation calls for the elimination of such conflicts.

To sum up, the architects of QM are to be credited with the creation of a flawless theory of atoms, molecules and the condensed state of matter, but the so-called "breakdown of classical physics" is a poor substitute for the actual separation of the classical theory into perennial and obsolete parts. The failure to remove the latter manifests itself as a "paradox" that was improperly attributed to a flaw of QM, whereas actually it is a flaw in the $20^{\text {th }}$ century interpretation of classical physics. Whereas this interpretation may have been problematic at the beginning of the $20^{\text {th }}$ century, after another century there should be no difficulty to handle the matter, assuming willingness to break with dogmatic thinking. The problem to be kept in mind is that Newton scholars have given well-deserved attention to the complications involved in the axiomatics for the mechanics of the Principia and to the paths that connect Newton with his precursors. By contrast, little attention is given to Newton as the founder of all of mathematical physics and to the imperfections the successors have only partially corrected in building late classical physics.

I have recently addressed this question of consolidation in Tisza: The reasonable effectiveness of mathematics in the natural sciences, Experimental Metaphysics, R. S. Cohen, et al. (eds.), The Kluwer Publ. Co.,1997, pp. 213-238. The title is an obvious takeoff on Wigner's paper and my aim was to recognize behind its playful ambivalence Wigner as the champion of reason who was biding his time. Although my paper is rather lengthy, the underlying ideas are relatively simple and I will sum up the main points.

The basic axiom of Newtonian mathematical physics is stated in the Preface to the first edition of the Principia: rational mechanics ought to address "motion" with the same precision as geometry handles the size and shape of idealized objects. It is interesting, however, that the core of Newton's method is also in line with Einstein's preference for "theories of principle". The association of "motion" with "mathematics" was a stroke of genius; but to appreciate its full impact, we must distinguish between the short-term and the long-term uses of this idea.

\section{From Newton...}

By producing the mechanics of the Principia Newton demonstrated that in the short-term there is a simple instance of motion, namely rigid translation that can be formalized in terms of available mathematics. Newton expected that his work would be effective also in the long-term. (The Third Rule of Reasoning in Philosophy, in Book III of the Principia. As an empiricist Newton added the Fourth Rule according to which this extrapolation is subject to experimental verification.)

It is a fact that the Third Rule exercised great attraction both for Einstein and Bohr. When Newtonian mechanics failed to account for the stability of the Rutherford atom, Einstein felt as if the ground were pulled from under him. Was this the end of the Newtonian epistemology? The epistemological discussions of the century are indeed marked by a tone of resignation. However looking back at the overall picture we see rather a vast explosion of knowledge. There was a retrenchment of expectations; but this is overshadowed by opening up new evolutionary avenues of exploration.

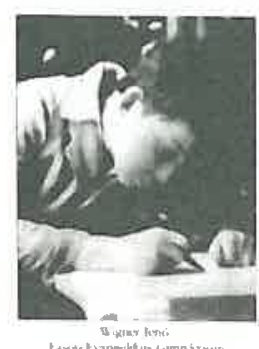

A century later we have every reason to bet on the evolutionary option. It is evident that the area of knowledge is being extended rather than narrowed. Instead of deploring the breakdown overcome only by revolution, we should understand the rules of the new evolution opened up by using new mathematics and wider scope of the concept of motion.

The first significant advance beyond Newton was directed at the expansion of the mathematical base. The work of continental mathematicians active over a century and a half converged into a highly successful analysis of the continuum. They considered Newtonian mechanics as a natural proving ground for the new mathematics. This was an expression of preference over the dynamics of Descartes but a rejection of Newton's Euclidean addiction; it led to an innovative analytical mechanics. The physics community accepted the innovation and called its Hamiltonian version canonical mechanics. This terminology implied, correctly, that a specific mathematical formalism might have a closer affinity to a theory of physics than alternative choices, even if one happens to be more familiar. Unfortunately, the term canonical is overstated; it is preferable to call the formalism optimal for Newtonian mechanics, but not to foreclose another choice for dealing with heat, light, electricity and magnetism, and for atomic physics.

The first one to break out of the canonical straight jacket was Faraday; he initiated an alternative approach, as all the listed nonmechanical phenomena can be also associated with the chemical structure of the atom. Maxwell's translation of Faraday's qualitative theory of the electromagnetic field into a mathematical formalism became an accepted branch of classical physics. This innovation was accepted by Einstein who stressed that the diversification of classical physics has to be met by consistency conditions. The requirement that Newtonian mechanics and Maxwellian electrodynamics be mutually consistent calls for the scrutiny of the concept of simultaneity and leads to the foundation of special relativity.

\section{... to Einstein}

Einstein emphasized that his method of transcending classical physics is logically flawless and he hoped that QM could be handled similarly. I suggest that this is a sensible program. Faraday's chemical departure goes much beyond the electromagnetic field and marks a bifurcation in the evolution of classical physics into a mechanical and a chemical branch that calls for the formulation of another consistency relation.

Whereas in case of SRT the sensitive concept was absolute time, in the case of QM there is a different particle concept implicit in Newtonian mechanics and in Faraday's chemical departure. Faraday's chemical atom was different from the mechanical atom implicit in Newtonian mechanics. The mechanical atom is defined by its position and velocity; it has no intrinsic structure. Two observations refer to the same particle if the observations refer to points on the same orbit as for the evening star and the morning star. By contrast, the chemical atom has intrinsic structure and 
different sample of the same structure form a class of indistinguishable particles, regardless of position.

Real particles have both mechanical and chemical properties and it is the uncertainty principle that ensures their mutual consistency. The Heisenberg principle restricts the scope of the mechanical measurement; the overall power of measurability is increased due to the emergence of the chemical branch. This becomes apparent in the context of the extraordinary junction in1859 when Bunsen and Kirchhoff joined chemical analysis with spectroscopic measurements. The discovery that all the stars are made of the same elements we find on earth was easily the largest extension of knowledge ever attained in a single step.

At exactly this time the concept of molecular mean free path was established which led to the kinetic gas theory. The bifurcation of physics into mechanical and chemical branches was firmly in place. Accordingly, it is misleading to speak of the breakdown of classical physics; we have to differentiate between the two branches. The fact that the application of Newtonian mechanics fails to explain the observed stability of a discrete set of states of the nuclear atom, indicates the failure of mechanical classical physics on the atomic level. By contrast, the Bunsen- Kirchhoff landmark indicates a great expansion of the scope of the chemical branch. It led through Planck to quantum theory and on to QM.

Although the bare facts of the discovery of spectrum analysis are well known, not enough is made of the epistemological revolution involved that marks a vast enrichment of the methods of acquiring knowledge.

I claim that such an epistemological principle is implicit in the Bunsen-Kirchhoff spectrum analysis. In this procedure light beams replaced the chemical reagents of traditional qualitative analysis. Light beams travel unimpeded though space and therefore the junction of chemical and optical methods vastly expand what these methods could achieve on their own. This is connected with an entirely new conception of measurement. It is very different from the Newtonian prediction that is vulnerable to any randomness. The light beam emerging from the diffraction grating consists of a random stream of photons yet leads to an accurate inference of the photon energy. This answers Einstein's concern that God does not play dice with us. He certainly does and to great advantage for us.

All this suggests that the chemical branch of classical physics should be given an increasing role in the formulation of the link between classical and quantum physics. This would call for giving chemical principles a greater role within the context of modern physics.

\section{... to Wigner}

Indeed, among the great scientific achievements of the $20^{\text {th }}$ century was the confluence of mechanics and chemistry, separated by a millennial tradition. In the words of J. of Wigner: "we can be proud of the unification of physics and chemistry that happened in our century." (See The Unity of Science I/1,1988, p 5, reprinted in Fizikai Szemle, 92, 1992, p. 436 ). This momentous event did not receive the appreciation it deserved. I suggest that this is because an ambiguity clouded the meaning of the term "unification". A widespread interpretation preferred reduction of chemistry to mechanics.

This expectation collapsed when Newtonian mechanics failed to account for the stability of the nuclear atom. What actually happened was a consistent joint use of the disciplines and the particle concept of QM is in closer harmony with chemistry than with mechanics.

I am glad to see that Hungarian education tends to unify basic chemical experience, such as the Periodic Table, quantum states, chemical bonds with fundamental atomic physics. (George Marx, Physics Education, September-November 1976, Institute of Physics, UK.)

\section{Remembering E. Wigner}

I wish to conclude on a personal note. I did not belong to Wigner's circle of intimate Hungarian friends, but our paths crossed since the beginning of my life in physics. I was a mathematics student in Budapest and Göttingen, where I attended Max Born's first ever course on quantum mechanics. I was impressed that higher mathematics found application to subtle empirical problems and-somewhat hesitantly-considered changing from mathematics to mathematical physics. This was the background when on vacation in Budapest I got an unexpected invitation for tea from Eugene Wigner. By that time, 1929, he was already a foremost theorist; the difference in our status was staggering. The invitation was an indication of the kindness and helpfulness of Eugene, for which he was well known. This invitation helped me overcome my timidity in making my important decision.

on vacation in To return to the present, beyond my limited personal contacts I was also Budapest | got engrossed with Wigner's style of insisting on the integrity of mathematics, no less than its empirical adequacy. I hope that this affinity ensures my credentials to interpret an unexpected invitation for tea. an aspect of his work that he left ambiguous. Traditional principles refuted by experiment must be abandoned. This does not call for a revolutionary break with the past. At this late stage tradition is likely to be only slightly out of focus, the flaw of tradition is to be removed by careful analysis. The required methods were not available at the turn of the $20^{\text {th }}$ century and the empirically all but perfect QM seemed paradoxical from the perspective of an obsolete tradition. We ought to remove the paradoxes of QM not by changing this theory, but by removing the obsolete part of classical physics. From my familiarity with Wigner's work, I feel that his understated alternative of pursuing C. S. Peirce's "secret" comes closer to his real views than the apparently flippant endorsement of the freewheeling use of paradoxes to support subjective beliefs. If $\mathrm{my}$ analysis of the situation would in any way contribute to a critical review of ancient preconceptions, I am confident that Eugene would endorse my role as his mouthpiece.

\section{Acknowledgements}

I wish to thank Abner Shimony for discussions, particularly on the background in which the paper in question came about. Wigner thanks him for the reference to Peirce, but Abner tells me that the selection of the motto was Wigner's. It was important to him. I also thank George Marx for informing me about current trends in Hungarian education. 